\title{
A Reliable Approach for Modeling the Actual Antenna Pattern in Millimeter-Wave Communication
}

\author{
Fulvio Babich and Massimiliano Comisso
}

\begin{abstract}
4 Abstract-This letter presents a mathematical framework for 5 evaluating the link capacity of an interfered communication link 6 in a millimeter-wave mobile scenario, accounting in detail for the 7 shape of the antenna pattern and for the statistic of the direction 8 of arrivals. The developed approach, whose accuracy is verified by 9 Monte Carlo validations in $2 \mathrm{D}$ and 3D wireless environments, does 10 not require simplified antenna models, thus enabling to maintain 11 the actual pattern during the analysis.
\end{abstract}

12 Index Terms-Millimeter-wave communication, antenna radia13 tion pattern, interference, direction of arrival, link capacity.

\section{INTRODUCTION}

${ }_{17}^{16} \mathbf{T}$ HE growing interest towards millimeter-wave (mmWave) technology has lead to the development of several propos18 als [1]-[8], for analyzing and improving the functionalities en19 abled by the IEEE 802.15.3c and 802.11ad standards [9], [10]. 20 Within these proposals, the antenna pattern plays a fundamental 21 role, since the small size of the radiating elements operating in 22 the $60 \mathrm{GHz}$ band has made feasible the use of antenna arrays 23 on the communication devices, thus enabling spatial reuse by 24 beamforming operations [11]. However, to maintain the ana25 lytical tractability of a considered problem, the pattern shape 26 is often simplified. The most common pattern approximations 27 adopt circular sectors [1], infinitesimal beamwidths [2], flat28 topped geometries [3]-[5], and directional models [6]-[8].

29 These approximations idealize many aspects of a real pattern, 30 whose shape may be in general much more complex than that 31 assumed in the commonly adopted simplified models. In fact, 32 this shape may include transition zones and ripples in the main 33 lobe, grating and secondary lobes, and narrow or large null 34 regions [12]. The difference between ideal and actual pattern 35 may introduce considerable discrepancies between theoretical 36 and practical results. Therefore, a more realistic pattern may be 37 introduced to improve the reliability of the analysis. This adop38 tion however makes difficult to derive closed-form expressions 39 for an investigated link quality metric, such as, for example, 40 the success probability. Thus, a modeling strategy capable to 41 guarantee the analytical tractability of a considered problem 42 maintaining the actual shape of the pattern during the analysis 43 may represent a desirable advance.

44 To deal with this issue, this letter proposes a theoretical 45 approach for an accurate modeling of the antenna pattern in 2D 46 and 3D scenarios. The approach is applied to a problem of link 47 capacity estimation in an interfered multipath-fading environ48 ment. Monte Carlo validations are used to verify the accuracy

Manuscript received April 1, 2015; revised June 12, 2015; accepted June 12, 2015. The associate editor coordinating the review of this paper and approving it for publication was T. Yioultsis.

The authors are with the Dipartimento di Ingegneria e Architettura (DIA), University of Trieste, 34100 Trieste, Italy (e-mail: mcomisso@units.it).

Digital Object Identifier 10.1109/LCOMM.2015.2445764 of the developed framework, which furthermore provides, in 49 some specific cases, expressions available in analytical form. 50

The letter is organized as follows. Section II introduces the 51 network scenario. Section III presents the pattern modeling 52 approach and the capacity analysis. Section IV discusses the 53 numerical results. Section V summarizes the main conclusions. 54

\section{SCEnARIO}

Consider a wireless network in which a destination D is 56 located at the center $O$ of a ball $B_{v}(O, \bar{R})$ of radius $\bar{R}$ and 57 dimension $v \in\{2,3\}$. This destination communicates with its 58 desired source $\mathrm{S}$ lying at a distance $\hat{R}(\leq \bar{R})$ in the presence of $L 59$ interferers uniformly distributed in $B_{v}(O, \bar{R})$, that is, on a disk 60 for $v=2$, and in a ball for $v=3$. Accordingly, the cumulative 61 density function (cdf) of the distance $R_{l}$ between $\mathrm{D}$ and the $l$-th 62 interferer may be expressed as [13]:

$$
F_{R_{l}}\left(r_{l}\right)=\frac{1}{\bar{R}^{v}} \begin{cases}0 & r_{l}<0 \\ r_{l}^{\nu} & 0 \leq r_{l} \leq \bar{R} \\ \bar{R}^{v} & r_{l}>\bar{R}\end{cases}
$$

In reception, $\mathrm{D}$ adopts a normalized antenna power gain pattern 64 $\mathcal{P}(\omega)$, which is obtained from a broadside array and where 65 the direction $\omega$ is the azimuth angle $\phi \in \boldsymbol{\Omega}_{2}=[0,2 \pi)$ for $\nu=66$ 2 , and the zenith-azimuth pair $(\theta, \phi) \in \boldsymbol{\Omega}_{3}=[0, \pi] \times[0,2 \pi) 67$ for $v=3$. In particular, a uniform linear array (ULA) of $N 68$ elements lying on the $x$-axis is adopted for $v=2$, while a 69 uniform square array (USA) of $N \times N$ elements lying on the 70 $x-z$ plane is adopted for $v=3$. Thus, to jointly model the 2D 71 and $3 \mathrm{D}$ cases, $\mathcal{P}(\omega)$ may be represented by [12]:

$$
\mathcal{P}(\omega)=\prod_{j=1}^{\nu-1}\left|\frac{\sin \left[N \pi d S_{j}^{(\nu)}(\omega)\right]}{N \sin \left[\pi d S_{j}^{(\nu)}(\omega)\right]}\right|^{2},
$$

where $d$ is the inter-element distance expressed as a multiple of 73 the carrier wavelength $\lambda$, and:

$$
S_{j}^{(\nu)}(\omega)= \begin{cases}\cos \phi & j=1, v=2 \\ \sin \theta \cos \phi & j=1, v=3 \\ \cos \theta & j=2, v=3\end{cases}
$$

The propagation channel is characterized by path-loss attenua- 75 tion and multipath-fading. More precisely, the path-loss func- 76 tion is:

$$
\varrho\left(r_{l}\right)=\left(r_{l}^{\alpha}+\epsilon\right)^{-1},
$$

where $\alpha(>2)$ is the path-loss exponent and $\epsilon$ is a nonnegative 78 parameter that identifies a classic unbounded path-loss model 79 for $\epsilon=0$ and a bounded path-loss model for $\epsilon>0$. For each 80 source (desired or interfering), a probability density function 81 (pdf) $f_{Q}(q)$ models the power fluctuation $Q$ due to fading, and 82 
83 another pdf $f_{\Omega^{\prime}}\left(\omega^{\prime}\right)$ models the spreading of the direction of 84 arrival (DOA) $\Omega^{\prime}$ due to multipath. More precisely, $f_{\Omega^{\prime}}\left(\omega^{\prime}\right)$ 85 is selected as a Laplacian distribution, whose reliability in 86 describing the spatial channel has been assessed by several 87 measurement campaigns [14]. Hence, the pdf of the DOA may 88 be expressed for $v \in\{2,3\}$ as [14]:

$$
f_{\Omega^{\prime}}\left(\omega^{\prime}\right)= \begin{cases}K_{\nu} \exp \left(-\left|\rho^{(\nu)}\left(\omega^{\prime}\right)\right|\right) & \omega^{\prime} \in \boldsymbol{\Omega}_{v} \\ 0 & \text { otherwise }\end{cases}
$$

89 where $K_{v}$ is a normalization constant, and:

$$
\rho^{(v)}\left(\omega^{\prime}\right)=\left(\frac{2}{\sigma^{2}}\right)^{\frac{v-1}{2}} \cdot \begin{cases}\phi-\pi & v=2 \\ (\theta-\pi / 2)(\phi-\pi) & v=3\end{cases}
$$

90 with $\sigma$ denoting the angular spread. For $v=3$, (5) is modeled 91 as the product of two univariate Laplacian pdfs, thus assuming, 92 as in [14], the separability of the zenith and azimuth statistics.

\section{ANALYSIS}

94 The analysis of the described scenario requires two steps. 95 The first one provides a probability mass function (pmf) of 96 an equivalent gain that jointly models $\mathcal{P}(\omega)$ and $f_{\Omega^{\prime}}\left(\omega^{\prime}\right)$. The 97 second step provides a parametric family of link capacity 98 values, each obtained for a given gain, which are subsequently 99 weighed according to the pmf, so as to evaluate the overall link 100 capacity by applying the concept of mixture distribution [15].

\section{A. Pattern-DOA Statistic Modeling}

102 As a first step, one has to derive the equivalent pattern [16]:

$$
g(\omega)=\int_{\mathbf{\Omega}_{v}} \mathcal{P}\left(\omega^{\prime}\right) f_{\Omega^{\prime}}\left(\omega^{\prime}-\omega\right) \mathrm{d} \omega^{\prime},
$$

103 which enables to jointly account for the receiving pattern and 104 the DOA statistic within a unique function by averaging each 105 value of $\mathcal{P}(\omega)$ over the pdf $f_{\Omega^{\prime}}\left(\omega^{\prime}\right)$. Since $\omega$ is the realization 106 of a random variable (r.v.) $\Omega$, also the equivalent gain may 107 be viewed as a r.v. $G$. In general, $\mathcal{P}(\omega)$, and hence $g(\omega)$, are 108 not invertible functions of $\omega$, thus the statistic of $G$ cannot 109 be derived in closed-form, and an approximated strategy must 110 be developed. To this aim, one may recall that $\mathcal{P}(\omega)$ is a 111 normalized pattern and that $f_{\Omega^{\prime}}\left(\omega^{\prime}\right)$ is a pdf, thus the values 112 of $g(\omega)$ belong to the interval $[0,1]$. This interval may be 113 subdivided into $M-1$ adjacent subintervals of equal length $114 \chi=1 /(M-1)$, to obtain the set of points $\mathbf{G}=\left\{g_{i}: g_{i}=(i-\right.$ $1151) \chi, i=1, \ldots, M\}$. The number of directions towards which 116 the equivalent gain is equal to $g_{i}$ may be evaluated as $\#\left(\boldsymbol{\Xi}_{i}\right)$, 117 which denotes the cardinality of the set $\boldsymbol{\Xi}_{i}=\left\{\omega: g(\omega)=g_{i}\right\}$. 118 Therefore, observing that $\boldsymbol{\Xi}_{1}, \ldots, \boldsymbol{\Xi}_{M}$ is a sequence of disjoint 119 sets, the pmf of $G$ may be estimated as:

$$
f_{G}\left(g_{i}\right)=\frac{\#\left(\boldsymbol{\Xi}_{i}\right)}{\sum_{i=1}^{M} \#\left(\boldsymbol{\Xi}_{i}\right)}, \quad g_{i} \in \mathbf{G} .
$$

120 The proposed pattern modeling approach has the considerable 121 advantage of modeling $\mathcal{P}(\omega)$ and $f_{\Omega^{\prime}}\left(\omega^{\prime}\right)$ by a unique r.v., whose 122 accuracy in describing $g(\omega)$ may be controlled through the 123 number of gain samples $M$, which may be increased to allow 124 a more reliable modeling of the actual statistic of $g(\omega)$.

\section{B. Link Capacity}

Once the pmf of $g(\omega)$ is available, the interference analysis 126 may be carried out for a given value $g_{i}$, that is, assuming the 127 equivalent gain as constant. To this purpose, using (4), the 128 power received by the destination D from the $l$-th interferer for 129 a gain $g_{i}$ in absence of mobility may be expressed as:

$$
T_{l}=k g_{i} \varrho\left(R_{l}\right)=k g_{i}\left(R_{l}^{\alpha}+\epsilon\right)^{-1},
$$

where $k$ is a constant accounting for the height of the antennas 131 and for the transmission power (assumed equal for all sources). 132 The cdf of $T_{l}$ for a given $g_{i}$ may be evaluated by inverting (9) 133 with respect to $R_{l}$ and using (1), thus obtaining:

$$
F_{T_{l}}\left(t_{l} ; g_{i}\right)= \begin{cases}0 & t_{l}<g_{i} \varsigma_{1} \\ 1-\frac{1}{\bar{R}^{\nu}}\left(\frac{k g_{i}}{t_{l}}-\epsilon\right)^{\beta} & g_{i} \varsigma_{1} \leq t_{l} \leq g_{i} \varsigma_{2} \\ 1 & t_{l}>g_{i} \varsigma_{2}\end{cases}
$$

where $\beta=v / \alpha, \varsigma_{1}=k \varrho(\bar{R})$, and $\varsigma_{2}=k \varrho(0)$. Recalling that 135 $f_{Q}(q)$ is the pdf of $Q$ that models the fading effects, the cdf of 136 $P_{l}=T_{l} Q$ may be derived from the product distribution [13]:

$$
F_{P_{l}}\left(p_{l} ; g_{i}\right)=\int_{0}^{+\infty} f_{Q}(q) F_{T_{l}}\left(\frac{p_{l}}{q} ; g_{i}\right) \mathrm{d} q,
$$

which can be exploited to obtain the statistic of the total inter- 138 ference $I$. Usually, the pdf of $I$ cannot be evaluated in closed- 139 form, and hence approximations based on the nearest interferers 140 have been introduced [2], [3]. In particular, according to [2], I 141 may be usefully approximated by:

$$
I=\sum_{l=1}^{L} P_{l} \approx H_{L}^{(1 / \beta)} \max \left\{P_{1}, \ldots, P_{L}\right\},
$$

where $H_{L}^{(1 / \beta)}=\sum_{l=1}^{L} l^{-1 / \beta}$ is the generalized harmonic num- 143 ber of order $L$ in power $1 / \beta$. Using the relationship for the 144 scaling of r.v.s [13], the cdf and the pdf of $I$ for a given $g_{i}$ may 145 be hence calculated, respectively, from:

$$
\begin{aligned}
F_{I}\left(p ; g_{i}, L\right) & \cong\left[F_{P_{l}}\left(\frac{p}{H_{L}^{(1 / \beta)}} ; g_{i}\right)\right]^{L}, \\
f_{I}\left(p ; g_{i}, L\right) & =\frac{\mathrm{d} F_{I}}{\mathrm{~d} p} .
\end{aligned}
$$

Recalling that $\hat{R}$ denotes the S-D distance and that the maxi- 147 mum of $g(\omega)$ is steered towards $S$, the desired signal power may 148 be expressed as $\Delta=\hat{t} Q$, where $\hat{t}=k \max \{g(\omega)\} \varrho(\hat{R})$. Hence, 149 the corresponding pdf is $f_{\Delta}(\delta)=f_{Q}(\delta / \hat{t}) / \hat{t}$. This latter statistic 150 enables to obtain the cdf of the signal-to interference ratio (SIR) 151 $\Upsilon=\Delta / I$ from the ratio distribution [13]:

$$
F_{\Upsilon}\left(v ; g_{i}, L\right)=1-\frac{1}{\hat{t}} \int_{0}^{+\infty} f_{Q}\left(\frac{\delta}{\hat{t}}\right) F_{I}\left(\frac{\delta}{v} ; g_{i}, L\right) \mathrm{d} \delta .
$$

The result of the S-D communication may be then established 153 adopting a SIR threshold $\psi$ that accounts for modulation, 154 coding, packet length, and required packet error rate [4], [8]. 155 Thus, the capture probability for a given $g_{i}$ is evaluated as:

$$
\eta\left(\psi ; g_{i}, L\right)=\operatorname{Pr}\{v>\psi\}=1-F_{\Upsilon}\left(\psi ; g_{i}, L\right) .
$$


157 It is interesting to observe that, in the presence of Rayleigh 158 fading, the pdf of $I$ and the capture probability for $L=1$ may 159 be represented in analytical form. To this aim, evaluating (11) 160 for $f_{Q}(q)=e^{-q} u(q)$, where $u(\cdot)$ is the Heaviside step function 161 with $u(0)=0$, the cdf of $P_{l}$ may be derived as:

$$
F_{P_{l}}\left(p_{l} ; g_{i}\right)=\int_{\frac{p_{l}}{g_{i} \varsigma_{2}}}^{\frac{p_{l}}{g_{i} \varsigma_{1}}} e^{-q}\left[1-\frac{1}{\bar{R}^{v}}\left(\frac{k g_{i} q}{p_{l}}-\epsilon\right)^{\beta}\right] \mathrm{d} q+\int_{0}^{\frac{p_{l}}{g_{i} \varsigma_{2}}} e^{-q} \mathrm{~d} q,
$$

162 which, recalling that $\varsigma_{1}=k /\left(\bar{R}^{\alpha}+\epsilon\right)$ and $\varsigma_{2}=k / \epsilon$, provides $163(18)$, shown at the bottom of the page, where $\gamma(\cdot, \cdot)$ is the lower 164 incomplete gamma function [17]. Now, using (18) in (13)-(16), 165 one may then obtain $f_{I}\left(p ; g_{i}, L\right)$ in $(19)$ and $\eta\left(\psi ; g_{i}, 1\right)$ in (20), 166 also, shown at the bottom of the page, where $\zeta_{L, \beta}=k H_{L}^{(1 / \beta)}$ 167 and ${ }_{2} F_{1}(\cdot, \cdot ; \cdot ; \cdot)$ is the hypergeometric function [17].

168 The capacity of the S-D link may be finally evaluated. To 169 this purpose, the parametric family $\eta\left(\psi ; g_{i}, L\right)$, resulting for $i=$ $1701, \ldots, M$, is employed to derive the capture probability from 171 the mixture distribution [15]:

$$
\eta(\psi ; L)=\sum_{i=1}^{M} \eta\left(\psi ; g_{i}, L\right) f_{G}\left(g_{i}\right)
$$

172 This latter expression may be used to estimate the limiting 173 performance of the interfered link according to the Shannon 174 bound, thus obtaining the link capacity as:

$$
C(\psi ; L)=\eta(\psi ; L) \log _{2}(1+\psi) .
$$

175 It is useful to notice that the proposed pattern modeling ap176 proach allows one to account for the impact of $\mathcal{P}(\omega)$ and $f_{\Omega^{\prime}}\left(\omega^{\prime}\right)$ 177 not only on the link capacity, but on any quantity for which a 178 parametric family of pdfs or cdfs has been calculated during 179 the analysis. For example, one may evaluate, again from the 180 mixture distribution, the pdf of the interference power as:

$$
f_{I}(p ; L)=\sum_{i=1}^{M} f_{I}\left(p ; g_{i}, L\right) f_{G}\left(g_{i}\right)
$$

181 Observe that the expressions in (21)-(23) together with those in 182 (18)-(20) represent a significant result, since, even if the latter 183 ones may seem formally elaborated, they have two relevant 184 advantages that are uncommonly present at the same time in 185 theoretical mmWave modeling: the maintenance of the actual 186 pattern as it is, and the availability of analytical forms.

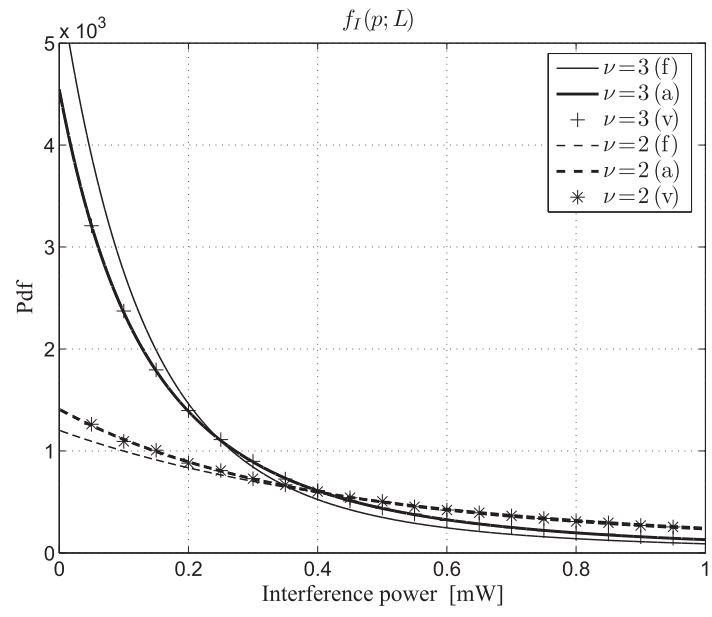

Fig. 1. Pdf of the interference in 2D and 3D cases for $\bar{R}=2 \hat{R}=10 \mathrm{~m}, \epsilon=1$, $L=1$ (f: flat-topped pattern, a: actual pattern, v: Monte Carlo validation).

\section{RESULTS}

The results are evaluated for $N=4, d=1 / 4, \alpha=3, \sigma=188$ $\pi / 3, \chi=0.01, k=1$ in a Rayleigh fading scenario, and are 189 verified by Monte Carlo validations. Each analytical curve 190 obtained from the actual pattern is compared with that obtained 191 from a flat-topped pattern [3]-[5], in order to check the sensi- 192 tivity of the results on the actual pattern details. According to 193 the typical rules adopted to derive the flat-topped model of a 194 given pattern, the flat-topped pattern is generated so as to have 195 the main lobe width equal to the half-power beamwidth $\Omega_{3 \mathrm{~dB}} 196$ of the actual pattern, the main lobe gain equal to the mean gain 197 of the actual pattern inside $\Omega_{3 \mathrm{~dB}}$, and the back-lobe gain equal 198 to the mean gain of the actual pattern outside $\Omega_{3 \mathrm{~dB}}$.

199

Figs. 1 and 2 report the pdf of the interference power and the 200 link capacity, respectively, for $\bar{R}=2 \hat{R}=10 \mathrm{~m}, \epsilon=1, L=1.201$ The figures refer to the 2D case for a ULA of $N=4$ elements, 202 and to the $3 \mathrm{D}$ case for a USA of $N \times N=4 \times 4$ elements. The 203 significant matching between the analysis obtained from the 204 actual pattern and the validation confirms the accuracy of 205 the developed framework. Furthermore, this matching reveals 206 that the characteristics of the actual pattern not included in its 207 flat-topped model may have, mainly in the 3D case, a not negli- 208 gible influence on the final results. This aspect is confirmed by 209 Fig. 3, which is obtained for $\nu=3, \bar{R}=2 \hat{R}=10 \mathrm{~m}, \epsilon=1$, and 210 different $L$ values, and by Fig. 4, which is obtained for $v=3,211$ $\bar{R}=2 \hat{R}=1 \mathrm{~m}, L=1$, and different $\epsilon$ values. With reference 212 to this latter figure, which shows the critical impact of the 213

$$
\begin{aligned}
& F_{P_{l}}\left(p_{l} ; g_{i}\right)=\left\{1-\exp \left(-\frac{\epsilon p}{k g_{i}}\right)\left[\exp \left(-\frac{\bar{R}^{\alpha} p}{k g_{i}}\right)+\left(\frac{k g_{i}}{\bar{R}^{\alpha} p}\right)^{\beta} \gamma\left(1+\beta, \frac{\bar{R}^{\alpha} p}{k g_{i}}\right)\right]\right\} u\left(p_{l}\right) \\
& f_{I}\left(p ; g_{i}, L\right) \cong F_{P_{l}}^{L-1}\left(\frac{k p}{\zeta_{L, \beta}} ; g_{i}\right) \exp \left(-\frac{\epsilon p}{g_{i} \zeta_{L, \beta}}\right)\left[\exp \left(-\frac{\bar{R}^{\alpha} p}{g_{i} \zeta_{L, \beta}}\right) \frac{\epsilon L}{g_{i} \zeta_{L, \beta}}+\frac{L\left(g_{i} \beta \zeta_{L, \beta}+\epsilon p\right)}{\left(g_{i} \zeta_{L, \beta}\right)^{1-\beta} p^{1+\beta} \bar{R}^{v}} \gamma\left(1+\beta, \frac{\bar{R}^{\alpha} p}{g_{i} \zeta_{L, \beta}}\right)\right] u(p) \\
& \eta\left(\psi ; g_{i}, 1\right)= \begin{cases}1-\beta k g_{i} \psi\left[\frac{\pi\left(\epsilon \hat{t}+k g_{i} \psi\right)^{\beta-1}}{\hat{t}^{\beta} \bar{R}^{\nu} \sin (\beta \pi)}-\frac{1}{(1-\beta) \hat{t}^{\alpha}}{ }_{2} F_{1}\left(1,1-\beta ; 2-\beta ;-\frac{\epsilon \hat{t}+k g_{i} \psi}{\hat{t}^{\alpha}}\right)\right] & 0<\beta<3 / 2, \beta \neq 1 \\
1-\frac{k g_{i} \psi}{\hat{t} \bar{R}^{\alpha}} \log \left(1+\frac{\hat{T} \bar{R}^{\alpha}}{\epsilon \hat{t}+k g_{i} \psi}\right) & \beta=1\end{cases}
\end{aligned}
$$




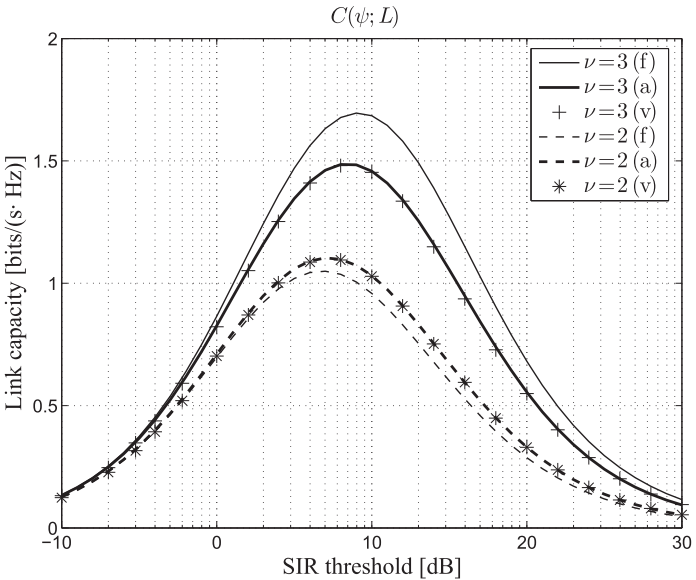

Fig. 2. Link capacity in $2 \mathrm{D}$ and $3 \mathrm{D}$ cases for $\bar{R}=2 \hat{R}=10 \mathrm{~m}, \epsilon=1, L=1$ (f: flat-topped pattern, a: actual pattern, v: Monte Carlo validation).

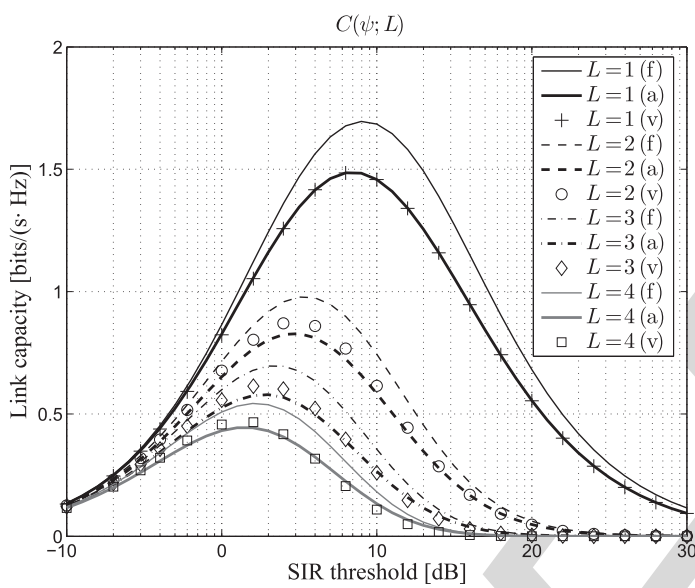

Fig. 3. Link capacity in 3D case for $\bar{R}=2 \hat{R}=10 \mathrm{~m}, \epsilon=1$, and different $L$ values (f: flat-topped pattern, a: actual pattern, v: Monte Carlo validation).



Fig. 4. Link capacity in $3 \mathrm{D}$ case for $\bar{R}=2 \hat{R}=1 \mathrm{~m}, L=1$, and different $\epsilon$ values (f: flat-topped pattern, a: actual pattern, v: Monte Carlo validation).

214 path-loss model in a short-range scenario, it is worth to no215 tice that the far-field assumption is satisfied. In fact, consid216 ering half-wavelength radiators and $d=1 / 4$, the maximum 217 dimension of the adopted USA of $N \times N$ elements is $D=$ $218 \sqrt{2}[N \lambda / 2+(N-1) \lambda / 4]$, with $\lambda=5 \mathrm{~mm}$ at $60 \mathrm{GHz}$. Thus, since the far-field region begins at $2 D^{2} / \lambda \cong 15 \mathrm{~cm}$ [12], the 219 far-field assumption may be considered satisfied. In summary, 220 the presented results suggest that the proposed approach, com- 221 bining the concepts of equivalent pattern and mixture distribu- 222 tion, may represent a useful support for the analysis of some 223 scenarios in which, even in the presence of other relevant 224 phenomena, such as interference, path-loss attenuation, and 225 multipath-fading, an investigated link quality metric turns out 226 to be sensitive to the actual pattern details.

\section{Conclusion}

A mathematical approach for modeling the DOA statistic and 229 the antenna pattern in 2D and 3D mmWave scenarios has been 230 presented and exploited to derive the link capacity in an inter- 231 fered multipath-fading environment. Monte Carlo validations 232 have confirmed the accuracy of the developed method, which 233 enables to properly account for the actual pattern shape, and, 234 in some specific cases, provides analytical expressions for the 235 investigated performance figures.

\section{REFERENCES}

[1] Q. Chen, J. Tang, D. T. C. Wong, X. Peng, and Y. Zhang, "Direc- 238 tional cooperative MAC protocol design and performance analysis for 239 IEEE 802.11ad WLANs," IEEE Trans. Veh. Technol., vol. 62, no. 6, 240 pp. 2667-2677, Jul. 2013.

[2] F. Babich and M. Comisso, "Capture analysis of mobile multi-packet 242 networks adopting spatial reuse: An alternative study," in Proc. IEEE 243 GLOBECOM, 2014, pp. 3477-3482.

[3] A. M. Hunter, J. G. Andrews, and S. Weber, "Transmission capacity of 245 ad hoc networks with spatial diversity," IEEE Trans. Wireless Commun., 246 vol. 7, no. 12, pp. 5058-5071, Dec. 2008.

[4] T. Bai and R. W. Heath, Jr., "Coverage and rate analysis for millimeter- 248 wave cellular networks," IEEE Trans. Wireless Commun., vol. 14, no. 2, 249 pp. 1100-1114, Feb. 2015.

[5] M. Kim, S.-E. Hong, and J. Kim, "Analysis of directional communication 251 via relaying devices in mmWave WPANs," IEEE Commun. Lett., vol. 16, 252 no. 3, pp. 342-345, Mar. 2012.

[6] Y. Wang, X. Kang, H. K. Garg, M. Motani, and Q. Chen, "Throughput 254 maximization for $60 \mathrm{GHz}$ WPANs via device cooperation," IEEE 255 Commun. Lett., vol. 18, no. 5, pp. 785-788, May 2014.

[7] C.-S. Sum and H. Harada, "Scalable heuristic STDMA scheduling 257 scheme for practical multi-Gbps millimeter-wave WPAN and WLAN 258 systems," IEEE Trans. Wireless Commun., vol. 11, no. 7, pp. 2658-2669, 259 Jul. 2012.

[8] S. Singh, R. Mudumbai, and U. Madhow, "Interference analysis for highly 261 directional $60-\mathrm{GHz}$ mesh networks: The case for rethinking medium ac- 262 cess control," IEEE/ACM Trans. Netw., vol. 19, no. 5, pp. 1513-1527, 263 Oct. 2011.

[9] IEEE Std for High Rate WPANs MAC/PHY Specific. Amend. 2: Millimeter 265 Wave Based Alternative PHY Ext., IEEE 802.15.3c, Oct. 2009. 266

[10] IEEE Std for WLAN MAC/PHY Specific. Amend. 3: Enhanc. for Very High 267 Throughput in the $60 \mathrm{GHz}$ Band, IEEE 802.11ad, 2014.

[11] J. G. Andrews et al. "What will 5G be?" IEEE J. Sel. Areas Commun., 269 vol. 32, no. 6, pp. 1065-1082, Jun. 2014.

[12] C. A. Balanis, Antenna Theory: Analysis and Design. New York, NY, 271 USA: Wiley, 1997.

[13] R. D. Yates and D. J. Goodman, Probability and Stochastic Processes. 273 New York, NY, USA: Wiley, 1999.

[14] M. B. Knudsen and G. F. Pedersen, "Spherical outdoor to indoor power 275 spectrum model at the mobile terminal," IEEE J. Sel. Areas Commun., 276 vol. 20, no. 6, pp. 1156-1169, Aug. 2002.

[15] S. Frühwirth-Schnatter, Finite Mixture and Markov Switching Models. 278 New York, NY, USA: Springer-Verlag, 2006.

[16] F. Babich and M. Comisso, "Throughput and delay analysis of 802.11- 280 based wireless networks using smart and directional antennas," IEEE 281 Trans. Commun., vol. 57, no. 5, pp. 1413-1423, May 2009.

[17] M. Abramowitz and I. A. Stegun, Handbook of Mathematical Func- 283 tions with Formulas, Graphs, and Mathematical Tables, ser. National 284 Bureau of Standards: Applied Mathematics, Series 55. Gaithersburg, 285 MD, USA: National Bureau of Standards, 1964. 
AUTHOR QUERY

NO QUERY. 